\title{
The sine-Gordon model with integrable defects revisited
}

\author{
Jean Avan $^{a}$ and Anastasia Doikou ${ }^{b}$ \\ ${ }^{a}$ LPTM, Universite de Cergy-Pontoise (CNRS UMR 8089), \\ F-95302 Cergy-Pontoise, France \\ ${ }^{b}$ Department of Engineering Sciences, University of Patras, \\ GR-26500 Patras, Greece \\ E-mail: avan@u-cergy.fr, adoikou@upatras.gr
}

ABSTRACT: Application of our algebraic approach to Liouville integrable defects is proposed for the sine-Gordon model. Integrability of the model is ensured by the underlying classical $r$-matrix algebra. The first local integrals of motion are identified together with the corresponding Lax pairs. Continuity conditions imposed on the time components of the entailed Lax pairs give rise to the sewing conditions on the defect point consistent with Liouville integrability.

KEywords: Integrable Hierarchies, Integrable Field Theories 


\section{Contents}

1 Introduction 1

$\begin{array}{lll}2 & \text { Preliminaries } & 3\end{array}$

3 The sine-Gordon model with integrable defect 5

$\begin{array}{lll}4 & \text { Discussion } & 15\end{array}$

\section{Introduction}

We recently proposed [1] a fully algebraic picture for a description of a Liouville integrable defect. It was successfully exemplified in the case of the continuous non-linear Schrödinger model (NLS), inducing us to now extend this procedure to the situation of the sine Gordon model. It is worth noting that the investigation of integrable defects has been a quite challenging problem, and there is a wealth of relevant articles in recent years at both classical and quantum level [1]-[23, 24].

Let us first recall the general procedure. We restrict ourselves for the time being to the case of a single defect. It is based on the construction of a suitable continuous transfer matrix generating the Poisson-commuting Hamiltonians and their associated timecomponent $\mathbb{V}$ of the continuous Lax pair: The continuous monodromy matrix is built as a coaction:

$$
T(L,-L, \lambda)=T^{+}\left(L, x_{0}, \lambda\right) \tilde{L}(\lambda) T^{-}\left(x_{0},-L, \lambda\right)
$$

The $T^{ \pm}$matrices are the monodromies of the differential operator $d / d x+L(x)$ where $L$ is the continuous Lax matrix $L(x)$ associated to the specific model, and $\tilde{L}$ is the defect matrix. The continuous Lax matrix is assumed to obey a linear ultra-local Poisson algebra parametrized by a non-dynamical skew-symmetric $r$-matrix. The defect $\tilde{L}$ is parametrized by discrete dynamical variables initially assumed to be independent of the continuous variables in $L(x)$ ("off-shell" condition).

Note: it must be emphasized that dropping any or some of these restrictions considerably complicates the issue even of building a bulk monodromy matrix: see e.g. [25] regarding the problems related to non-local and/or skew symmetric $r$-matrices and $[26,28]$ for the issue of finding the quadratic Poisson structure "derived" from a linear dynamical $r$-matrix structure.

Within our restricted conditions the bulk monodromy operators then obey a wellestablished quadratic Poisson algebra [29].

$$
\left\{T_{a}(\lambda), T_{b}(\mu)\right\}=\left[r_{a b}(\lambda-\mu), T_{a}(\lambda) T_{b}(\mu)\right]
$$


The same Poisson algebra is obeyed by the equal-point monodromy matrices:

$$
\left\{T_{a}\left(L, x_{0}, \lambda\right), T_{b}\left(L, x_{0}, \mu\right)\right\}=\left[r_{a b}(\lambda-\mu), T_{a}\left(L, x_{0}, \lambda\right) T_{b}\left(L, x_{0}, \mu\right)\right]
$$

and

$$
\left\{T_{a}\left(x_{0},-L, \lambda\right), T_{b}\left(x_{0},-L, \mu\right)\right\}=\left[r_{a b}(\lambda-\mu), T_{a}\left(x_{0},-L, \lambda\right) T_{b}\left(x_{0},-L, \mu\right)\right] .
$$

Liouville integrability is ensured from asking that the defect matrix $\tilde{L}$ obeys the same quadratic Poisson algebra with the same $r$ matrix as the bulk-interval monodromy operators $T\left(x_{0},-L, \lambda\right)$ and $T\left(x_{0},-L, \lambda\right)$, thereby imposing a strong constraint on the Poisson structure of the dynamical variables parametrizing the defect. The Poisson-commuting hierarchy of Hamiltonians is then obtained from expansion in $\lambda^{-1}$ of the $l n$ of the trace of the monodromy matrix (1.1). Poisson commutation is formally guaranteed by the underlying quadratic Poisson structure [29].

The time components of the Lax pair are then computed. They are evaluated separately in the right bulk $\left[x_{0}, L\right]$ and the left bulk $\left[-L, x_{0}\right]\left(\operatorname{resp} . \mathbb{V}^{+}(x)\right.$ and $\left.\mathbb{V}^{-}(x)\right)$ and on the defect point - from left and right (resp. $\tilde{\mathbb{V}}^{+}\left(x_{0}\right)$ and $\left.\tilde{\mathbb{V}}^{-}\left(x_{0}\right)\right)$. It is then required that $\mathbb{V}^{( \pm)}\left(x_{0}^{ \pm}\right)=\tilde{\mathbb{V}}^{( \pm)}\left(x_{0}\right)$ in order to eliminate singular contributions arising in the zero curvature condition written from the explicit Lax pair. This translates into sewing conditions $\left\{C_{ \pm}^{(j)}\right\}$ across the defect relating the right and left values of the $(j-1)$ th derivatives of the fields by functions of lower derivatives and the defect parameters. Sewing conditions are thus derived as necessary conditions to allow identification of the Hamiltonian equations of motion derived directly from $H^{(i)}$, with the equations derived from the zero curvature condition of the Lax pair $\mathbb{U}, \mathbb{V}^{(i)}$. They thus act as "regularizations" in the well-known canonical $[29,30]$ procedure yielding $H^{(i)}$ and the associated $\mathbb{V}^{(i)}$ through the classical $r$ matrix and (at least formally) guarantee the consistency of this procedure in the occurrence of a point-like defect.

The sewing conditions must now be regarded as dynamical constraints of the system, which in particular requires that the sub-manifold of the sewing conditions $\left\{C_{ \pm}^{(i)}\right\}$ be invariant under the Hamiltonian action. This set of conditions reads:

$$
\left\{\mathcal{H}^{(i)}, C_{ \pm}^{(j)}\right\} \text { belongs to the ideal generated by } C_{ \pm}^{(i)} .
$$

An important remark is required here. In general the sewing conditions do not Poissonclose on each other and represent therefore second-class constraints. Such was indeed the case in the NLS model. In this case the reduced phase space must be endowed with a structure of Dirac brackets to become an actual symplectic manifold on which a Liouvilleintegrable system can be defined. We recall that the Dirac brackets read (in a synthetic formulation):

$$
\{f, g\}_{D B} \equiv\{f, g\}_{P B}+\sum_{a, b}\left\{f, C_{a}\right\} M_{a b}^{-1}\left\{C_{b}, g\right\}
$$

where $f, g$ are any functions of the dynamical variables; \{\}$_{D B}$ must be evaluated on the constrained manifold; $C_{a}$ denote the constraints and $M_{a b}$ is the matrix of Poisson brackets of the constraints. 
It is now obvious from (1.6) that:

- if any two conserved charges, initially constructed off-shell, Poisson-commute at least weakly on the constrained manifold: $\left\{H_{i}, H_{j}\right\} \approx 0$;

- and if any such conserved charge preserves the constraints: $\left\{H_{i}, C_{a}\right\} \approx 0$ on the constrained manifold, then one finds:

$$
\left\{H_{i}, H_{j}\right\}_{D B}=0,
$$

thereby guaranteeing Liouville integrability of the defect theory on the manifold of sewing constraints endowed with the consistent Dirac bracket.

This procedure will now be applied to the sine-Gordon (SG) model, for which we shall consider two distinct parametrizations of so-called type-II or dynamical defects defects (see also $[4,10,11])$. We must immediately emphasize that this model provides an example where the initial off-shell continuous "conserved" Hamiltonians do not Poisson commute, but will be shown to weakly Poisson-commute once the sewing conditions are implemented, thereby guaranteeing Liouville integrability of the reduced model. By contrast in the NLS case the continuous Hamiltonians strongly (i.e. off-shell) Poisson-commuted. As seen above this does not modify the conclusions on Liouville integrability on-shell.

Let us further comment on this potential breaking of Poisson-commutation for the offshell defect-plus-continuous Hamiltonians. The Poisson structure (1.2) guarantees at least formally the Liouville integrability of the system under consideration. Poisson commutation of the trace of the logarithm of the monodromy matrix is formally an obvious direct consequence of this quadratic $r$-matrix structure, but needs to be checked on any given example, particularly for continuous plus discrete theories. Indeed, the conserved quantities are explicitly obtained as coefficients of the expansion of the trace-log in formal series of the spectral parameter, whereas the Poisson brackets are expressed as distribution-valued objects (see the $\delta(x-y)$ terms). This superposition of formal series and distributions may lead to subtleties in the evaluation of the continuous contributions close to the defect point due to regularizations, and break formal integrability by some "classical anomaly".

\section{Preliminaries}

A starting point in the description of classical integrable lattice models is the existence of the Lax pair $\mathbb{U}, \mathbb{V}$. Define $\Psi$ as being a solution of the following set of equations (see e.g. [29])

$$
\begin{aligned}
& \frac{\partial \Psi}{\partial x}=\mathbb{U}(x, t, \lambda) \Psi \\
& \frac{\partial \Psi}{\partial t}=\mathbb{V}(x, t, \lambda) \Psi
\end{aligned}
$$

$\mathbb{U}, \mathbb{V}$ being in general $n \times n$ matrices with entries defined as functions of complex valued dynamical fields, their derivatives, and the complex spectral parameter $\lambda$. Compatibility 
of the two aforementioned equations (2.1), (2.2) gives rise to the zero curvature condition

$$
\dot{\mathbb{U}}(x, t)-\mathbb{V}^{\prime}(x, t)+[\mathbb{U}(x, t), \mathbb{V}(x, t)]=0,
$$

which provides the equations of motion of the system at hand.

As is well known the generating function of the local integrals of motion is given by the expression

$$
\mathcal{G}=\ln (\operatorname{tr} T(L,-L, \lambda))
$$

where the monodromy matrix $T$ is defined as,

$$
T(L,-L, \lambda)=P \exp \left\{\int_{-L}^{L} d x \mathbb{U}(x)\right\},
$$

It is in fact a limit when $x$ goes to $L$ of a matrix-type solution of (2.1) normalized to be $\nVdash$ at $-L$.

We now impose that the operator $\mathbb{U}$ satisfy the ultra-local Poisson structure described by the linear algebraic relations

$$
\left\{\mathbb{U}_{1}(x, \lambda), \mathbb{U}_{2}(y, \mu)\right\}=\left[r_{12}(\lambda-\mu), \mathbb{U}(x, \lambda)+\mathbb{U}_{2}(y, \mu)\right] \delta(x-y)
$$

It is then straightforward to show that $T$ satisfies the fundamental quadratic algebra:

$$
\left\{T_{1}(\lambda), T_{2}(\mu)\right\}=\left[r_{12}(\lambda-\mu), T_{1}(\lambda) T_{2}(\mu)\right] .
$$

$r_{12}(\lambda-\mu)$ is the so-called classical $r$-matrix assumed here to be a non-dynamical skewsymmetric solution of the classical Yang-Baxter equation.

We shall focus our investigation here on the sine-Gordon model. In this case the $\mathbb{U}$ operator of the Lax pair is a $2 \times 2$ matrix and is given by [31]:

$$
\mathbb{U}(x, t, u)=\frac{\beta}{4 i} \pi(x, t) \sigma^{z}+\frac{m u}{4 i} e^{\frac{i \beta}{4} \phi \sigma^{z}} \sigma^{y} e^{-\frac{i \beta}{4} \phi \sigma^{z}}-\frac{m u^{-1}}{4 i} e^{-\frac{i \beta}{4} \phi \sigma^{z}} \sigma^{y} e^{\frac{i \beta}{4} \phi \sigma^{z}}
$$

$u \equiv e^{\lambda}, \sigma^{x, y, z}$ are the $2 \times 2$ Pauli matrices, and the associated classical $r$-matrix in this case is given by the familiar form [31]:

$$
r(\lambda)=\frac{\beta^{2}}{8 \sinh \lambda}\left(\begin{array}{cc}
\frac{\sigma^{z}+1}{2} \cosh \lambda & \sigma^{-} \\
\sigma^{+} & \frac{-\sigma^{z}+1}{2} \cosh \lambda
\end{array}\right) .
$$

Stating that the Lax operator $\mathbb{U}$ satisfies the linear Poisson algebra (2.6) is equivalent to setting that $\phi, \pi$ are canonical conjugates, i.e.

$$
\{\phi(x), \pi(y)\}=\delta(x-y) .
$$

Let us now apply the generic defect construction to the sine-Gordon model. 


\section{The sine-Gordon model with integrable defect}

In the presence of an integrable defect the monodromy matrix of the field theory is modified (see also $[1,6,13]$ ), and takes the generic form

$$
\begin{aligned}
T(L,-L, \lambda) & =T^{+}\left(L, x_{0}^{+}, \lambda\right) \tilde{L}(\lambda) T^{-}\left(x_{0}^{-},-L, \lambda\right) \\
& =P \exp \left\{\int_{x_{0}^{+}}^{L} d x \mathbb{U}^{+}(x)\right\} \tilde{L}(\lambda) P \exp \left\{\int_{-L}^{x_{0}^{-}} d x \mathbb{U}^{-}(x)\right\}
\end{aligned}
$$

Assuming that the defect Lax matrix $\tilde{L}$ also satisfies the quadratic Poisson algebra (2.7) $T$ given in (3.1) also satisfies (2.7).

Type-IIa defect. A first consistent parametrization of an integrable defect of the socalled Type-II or dynamical will be considered in this section. The classical $\tilde{L}$ matrix takes the form (type-IIa)

$$
\tilde{L}(\lambda)=\left(\begin{array}{cc}
e^{\lambda} V-e^{-\lambda} V^{-1} & \bar{a} \\
a & e^{\lambda} V^{-1}-e^{-\lambda} V
\end{array}\right) .
$$

Requiring that $\tilde{L}$ satisfies the algebraic relation (2.7), one extracts the following Poisson relations between the defect fields:

$$
\begin{aligned}
& \{V, \bar{a}\}=\frac{\beta^{2}}{8} V \bar{a}, \\
& \{V, a\}=-\frac{\beta^{2}}{8} V a, \\
& \{\bar{a}, a\}=\frac{\beta^{2}}{4}\left(V^{2}-V^{-2}\right)
\end{aligned}
$$

From these Poisson brackets one naturally extracts a cyclic variable $C_{0}=V^{2}+V^{-2}+$ $\bar{a} a$ identified as the Casimir of a deformed $\mathfrak{s l}_{2}$. This variable Poisson-commute with all other dynamical quantities and can therefore be fixed to some particular value $c_{0}$. We shall nevertheless keep the redundant three-parameter expression for $\tilde{L}$ for reasons of form simplicity in the explicit expressions.

Our first aim is to express the term of order $u$ in $\mathbb{U}$ independently of the fields, after applying a suitable gauge transformation [29]

$$
T^{ \pm}(x, y, \lambda)=\Omega^{ \pm}(x) \tilde{T}^{ \pm}(x, y)\left(\Omega^{ \pm}(y)\right)^{-1}, \quad \Omega^{ \pm}=e^{\frac{i \beta}{4} \phi^{ \pm} \sigma^{z}}
$$

The gauge transformed operator $\tilde{\mathbb{U}}$ is expressed as:

$$
\tilde{\mathbb{U}}^{ \pm}(x, t, u)=\frac{\beta}{4 i} \mathfrak{f}^{ \pm} \sigma^{z}+\frac{m u}{4 i} \sigma^{y}-\frac{m u^{-1}}{4 i} e^{-\frac{i \beta}{2} \phi^{ \pm} \sigma^{z}} \sigma^{y} e^{\frac{i \beta}{2} \phi^{ \pm} \sigma^{z}}
$$

where we define

$$
\mathfrak{f}^{ \pm}(x, t)=\pi^{ \pm}(x, t)+\phi^{ \pm^{\prime}}(x, t)
$$


We consider the following convenient decomposition for $\tilde{T}$, as $|u| \rightarrow \infty[29]$,

$$
\tilde{T}^{ \pm}(x, y, \lambda)=\left(1+W^{ \pm}(x, \lambda)\right) e^{Z^{ \pm}(x, y, \lambda)}\left(1+W^{ \pm}(y, \lambda)\right)^{-1}
$$

$W^{ \pm}$is an off-diagonal matrix and $Z^{ \pm}$is purely diagonal. They are expanded as:

$$
W^{ \pm}=\sum_{k=0}^{\infty} \frac{W^{ \pm(k)}}{u^{k}}, \quad Z^{ \pm}=\sum_{k=-1}^{\infty} \frac{Z^{ \pm(k)}}{u^{k}}
$$

Note that $\tilde{T}$ naturally satisfies the gauged Lax equation:

$$
\frac{\partial \tilde{T}^{ \pm}}{\partial x}=\tilde{\mathbb{U}}^{ \pm}(x, \lambda) \tilde{T}^{ \pm}(x, y, \lambda)
$$

Inserting expressions (3.7), (3.8) in (3.9) one identifies the matrices $W^{ \pm(k)}$ and $Z^{ \pm(k)}$. More precisely, we end up with an equation for the off-diagonal matrix:

$$
\frac{\partial W^{ \pm}}{\partial x}+W^{ \pm} \tilde{\mathbb{U}}_{D}^{ \pm}-\tilde{\mathbb{U}}_{D}^{ \pm} W^{ \pm}+W^{ \pm} \tilde{\mathbb{U}}_{A}^{ \pm} W^{ \pm}-\mathbb{U}_{A}^{ \pm}=0
$$

where the indices $D, A$ denote the diagonal and anti-diagonal part of the Lax operator $\tilde{\mathbb{U}}^{ \pm}$. In the $2 \times 2$ case the above equations provide Riccati-type equations for the entries of $W^{ \pm}$:

$$
\frac{\partial W_{i j}^{ \pm}}{\partial x}+W_{i j}^{ \pm}\left(\tilde{\mathbb{U}}_{j j}^{ \pm}-\tilde{\mathbb{U}}_{i i}^{ \pm}\right)+\left(W_{i j}^{ \pm}\right)^{2} \tilde{\mathbb{U}}_{j i}^{ \pm}-\tilde{\mathbb{U}}_{i j}^{ \pm}=0, \quad i \neq j \in\{1,2\} .
$$

while the diagonal matrix, which provides essentially the integrals of motion as will become transparent in what follows, obeys the following Lax equation:

$$
\frac{\partial Z_{j j}^{ \pm}}{\partial x}=\tilde{\mathbb{U}}_{j j}^{ \pm}+\tilde{\mathbb{U}}_{j i}^{ \pm} W_{i j}^{ \pm}
$$

Similarities with corresponding equations emerging in $[14,23,24]$ from the inverse scattering point of view are apparent as expected, given that one essentially solves the same fundamental equations (2.1) $\left(W_{i j} \rightarrow \Gamma_{i j}\right)$. Of course Liouville integrability is guaranteed within the present approach by construction (at least formally), whereas in the methodology of $[14,23,24]$ only the conservation of the charges for a singled-out time-evolution is shown through the zero curvature condition i.e. explicit use of the equations of motion. We shall further comment on these issues later in the text, especially regarding the theory in the presence of defects.

It is sufficient for our purposes here to identify only the first few terms of the expansions. Indeed based on equation (3.9) we conclude (see also [29]):

$$
\begin{aligned}
& W^{ \pm(0)}=i \sigma_{1}, \quad W^{ \pm(1)}=-\frac{i \beta}{m} \mathfrak{f}^{ \pm}(x) \sigma_{1}, \\
& W^{ \pm(2)}=\frac{2 i \beta \mathfrak{f}^{ \pm^{\prime}}}{m^{2}} \sigma_{2}-i \sin \left(\beta \phi^{ \pm}\right) \sigma_{2}-\frac{\beta^{2}\left(\mathfrak{f}^{ \pm}\right)^{2}}{2 i m^{2}} \sigma_{1} .
\end{aligned}
$$


We also need to identify the diagonal elements $Z^{ \pm(n)}$. In particular from equation (2.1) we extract the following expressions:

$$
\begin{aligned}
& Z^{+(-1)}=-\frac{i m\left(L-x_{0}\right)}{4} \sigma_{3}, \quad Z^{-(-1)}=-\frac{i m\left(L+x_{0}\right)}{4} \sigma_{3} \\
& Z^{+(1)}=\frac{m}{4}\left(\begin{array}{lll}
-\int_{x_{0}^{+}}^{L} d x W_{21}^{+(2)}(x) & \\
& & \int_{x_{0}^{+}}^{L} d x W_{12}^{+(2)}(x)
\end{array}\right) \\
& -\frac{m}{4}\left(\begin{array}{rl}
-i \int_{x_{0}}^{L} d x e^{-i \beta \phi^{+}} & \\
& i \int_{x_{0}^{+}}^{L} d x e^{i \beta \phi^{+}}
\end{array}\right), \\
& Z^{-(1)}=\frac{m}{4}\left(\begin{array}{lll}
-\int_{-L}^{x_{0}^{-}} d x & W_{21}^{-(2)}(x) & \\
& & \int_{-L}^{x_{0}^{-}} d x W_{12}^{-(2)}(x)
\end{array}\right) \\
& -\frac{m}{4}\left(\begin{array}{rl}
-i \int_{-L}^{x_{0}^{-}} d x e^{-i \beta \phi^{-}} & \\
& i \int_{-L}^{x_{0}^{-}} d x e^{i \beta \phi^{-}}
\end{array}\right) .
\end{aligned}
$$

Notice that for $-i u \rightarrow \infty$ the leading contribution, in the expansion in powers of $u^{-1}$, comes from the $Z_{11}^{ \pm}$elements. This observation will be subsequently quite useful.

The first step in our investigation is the derivation of the associated local integrals of motion. In particular, the energy and momentum in the presence of defect will be explicitly derived. Let us first recall the generating function of the local integrals of motion

$$
\mathcal{G}(\lambda)=\ln \left[\operatorname{tr} T^{+}\left(L, x_{0}, \lambda\right) \tilde{L}\left(x_{0}, \lambda\right) T^{-}\left(x_{0}, L, \lambda\right)\right]
$$

Schwartz boundary conditions are imposed at the end point of the system $\pm L$. Recalling also the ansatz for the monodromy matrices we conclude for the generating function:

$$
\mathcal{G}(\lambda)=\ln \operatorname{tr}\left[e^{Z^{+}\left(L, x_{0}\right)}\left(1+W^{+}\left(x_{0}\right)\right)^{-1}\left(\Omega^{+}\left(x_{0}\right)\right)^{-1} \tilde{L}\left(x_{0}\right) \Omega^{-}\left(x_{0}\right)\left(1+W^{-}\left(x_{0}\right)\right) e^{Z^{-}\left(x_{0},-L\right)}\right]
$$

Choosing to consider the $-i u \rightarrow \infty$ behavior we take into account the leading contribution for the $Z_{11}^{ \pm}$terms, then the generating function takes the form:

$$
\mathcal{G}(\lambda)=Z_{11}^{+}+Z_{11}^{-}+\ln \left[\left(1+W^{+}\left(x_{0}\right)\right)^{-1}\left(\Omega^{+}\left(x_{0}\right)\right)^{-1} \tilde{L}\left(x_{0}\right) \Omega^{-}\left(x_{0}\right)\left(1+W^{-}\left(x_{0}\right)\right)\right]_{11}
$$

Expanding the latter expression in powers of $u^{-1}$ we obtain the following:

$$
\mathcal{G}(\lambda)=\sum_{m=0}^{\infty} \frac{I^{(m)}}{u^{m}} .
$$

Recalling now the expression for the generating function of integrals of motion we conclude that

$$
\begin{aligned}
I^{(1)}= & -\frac{m}{4 i} \int_{-L}^{x_{0}^{-}} d x\left(-\frac{\beta^{2}}{2 m^{2}} \mathfrak{f}^{-2}(x)+\cos \left(\beta \phi^{-}(x)\right)\right)-\frac{m}{4 i} \int_{x_{0}^{+}}^{L} d x\left(-\frac{\beta^{2}}{2 m^{2}} \mathfrak{f}^{+2}(x)+\cos \left(\beta \phi^{+}(x)\right)\right) \\
& +\frac{i}{\mathcal{D}}\left(e^{-\frac{i \beta}{4}\left(\phi^{+}\left(x_{0}\right)+\phi^{-}\left(x_{0}\right)\right)} \bar{a}-e^{\frac{i \beta}{4}\left(\phi^{+}\left(x_{0}\right)+\phi^{-}\left(x_{0}\right)\right)} a\right)+\frac{\beta}{2 m \mathcal{D}}\left(\mathfrak{f}^{+}\left(x_{0}\right)+\mathfrak{f}^{-}\left(x_{0}\right)\right) \mathcal{A}
\end{aligned}
$$


where we define:

$$
\begin{aligned}
& \mathcal{D}=e^{-\frac{i \beta}{4}\left(\phi^{+}\left(x_{0}\right)-\phi^{-}\left(x_{0}\right)\right)} V+e^{\frac{i \beta}{4}\left(\phi^{+}\left(x_{0}\right)-\phi^{-}\left(x_{0}\right)\right)} V^{-1}, \\
& \mathcal{A}=e^{-\frac{i \beta}{4}\left(\phi^{+}\left(x_{0}\right)-\phi^{-}\left(x_{0}\right)\right)} V-e^{\frac{i \beta}{4}\left(\phi^{+}\left(x_{0}\right)-\phi^{-}\left(x_{0}\right)\right)} V^{-1}
\end{aligned}
$$

If we now perform the same expansion for $\lambda \rightarrow-\infty$, we basically end up with a similar expression, by simply exploiting the fundamental symmetry of the monodromy matrix:

$$
T\left(u^{-1}, \phi, \pi, V, a, \bar{a}\right)=T\left(-u,-\phi, \pi, V^{-1}, a, \bar{a}\right) .
$$

More precisely, one (relatively easily... ) concludes that:

$$
\begin{aligned}
I^{(-1)}= & \frac{m}{4 i} \int_{-L}^{x_{0}^{-}} d x\left(-\frac{\beta^{2}}{2 m^{2}} \hat{\mathfrak{f}}^{-2}(x)+\cos \left(\beta \phi^{-}(x)\right)\right)-\frac{m}{4 i} \int_{x_{0}^{+}}^{L} d x\left(-\frac{\beta^{2}}{2 m^{2}} \hat{\mathfrak{f}}^{+2}(x)+\cos \left(\beta \phi^{+}(x)\right)\right) \\
& -\frac{i}{\mathcal{D}}\left(e^{\frac{i \beta}{4}\left(\phi^{+}\left(x_{0}\right)+\phi^{-}\left(x_{0}\right)\right)} \bar{a}-e^{-\frac{i \beta}{4}\left(\phi^{+}\left(x_{0}\right)+\phi^{-}\left(x_{0}\right)\right)} a\right)+\frac{\beta}{2 m \mathcal{D}}\left(\hat{\mathfrak{f}}^{+}\left(x_{0}\right)+\hat{\mathfrak{f}}^{-}\left(x_{0}\right)\right) \mathcal{A}
\end{aligned}
$$

where we define

$$
\hat{\mathfrak{f}}^{ \pm}(\phi, \pi)=\mathfrak{f}^{ \pm}(-\phi, \pi)
$$

Of course any (even functional) combination of the quantities $I^{(1)}, I^{(-1)}$ can be picked as one of the charges in involution. In particular the standard sine-Gordon Hamiltonian is defined as:

$$
\begin{aligned}
\mathcal{H}= & \frac{2 i m}{\beta^{2}}\left(I^{(1)}-I^{(-1)}\right) \\
= & \int_{-L}^{x_{0}^{-}} d x\left(\frac{1}{2}\left(\pi^{-2}(x)+\phi^{{ }^{\prime} 2}(x)\right)-\frac{m^{2}}{\beta^{2}} \cos \left(\beta \phi^{-}(x)\right)\right) \\
& +\int_{x_{0}^{+}}^{L} d x\left(\frac{1}{2}\left(\pi^{+2}(x)+\phi^{+^{\prime}}(x)\right)-\frac{m^{2}}{\beta^{2}} \cos \left(\beta \phi^{+}(x)\right)\right) \\
& -\frac{4 m}{\beta^{2} \mathcal{D}} \cos \frac{\beta}{4}\left(\phi^{+}\left(x_{0}\right)+\phi^{-}\left(x_{0}\right)\right)(\bar{a}-a)+\frac{2 i}{\beta \mathcal{D}}\left(\phi^{+^{\prime}}\left(x_{0}\right)+\phi^{-^{\prime}}\left(x_{0}\right)\right) \mathcal{A}
\end{aligned}
$$

and we also identify the sine-Gordon momentum as:

$$
\begin{aligned}
\mathcal{P}= & \frac{2 i m}{\beta^{2}}\left(I^{(1)}+I^{(-1)}\right) \\
= & \int_{-L}^{x_{0}^{-}} d x{\phi^{-}}^{\prime}(x) \pi^{-}(x)+\int_{x_{0}^{+}}^{L} d x{\phi^{+}}^{\prime}(x) \pi^{+}(x) \\
& +\frac{4 m i}{\beta^{2} \mathcal{D}} \sin \frac{\beta}{4}\left(\phi^{+}\left(x_{0}\right)+\phi^{-}\left(x_{0}\right)\right)(\bar{a}+a)+\frac{2 i}{\beta \mathcal{D}}\left(\pi^{+}\left(x_{0}\right)+\pi^{-}\left(x_{0}\right)\right) \mathcal{A} .
\end{aligned}
$$

Explicit computation of the Poisson bracket $\{\mathcal{H}, \mathcal{P}\}$ now yields a number of non-zero terms; we shall come back to this issue after deriving the sewing conditions in order to apply the Dirac bracket formalism advocated in the Introduction. 
The next step is the derivation of the time components of the associated Lax pairs. Expressions of the time component $\mathbb{V}$ of the Lax pair are known (see e.g. [29]). The generic expressions for the bulk left and right theories as well as the defect points are given as $[1,32]$ :

$$
\begin{aligned}
& \mathbb{V}^{+}(x, \lambda, \mu)=t^{-1}(\lambda) \operatorname{tr}_{a}\left(T_{a}^{+}(A, x, \lambda) r_{a b}(\lambda-\mu) T_{a}^{+}\left(x, x_{0}, \lambda\right) \tilde{L}_{a}\left(x_{0}, \lambda\right) T_{a}^{-}\left(x_{0},-A, \lambda\right)\right) \\
& \mathbb{V}^{-}(x, \lambda, \mu)=t^{-1}(\lambda) \operatorname{tr}_{a}\left(T_{a}^{+}\left(A, x_{0}, \lambda\right) \tilde{L}_{a}\left(x_{0}\right) T_{a}^{-}\left(x_{0}, x, \lambda\right) r_{a b}(\lambda-\mu) T_{a}^{-}(x,-A, \lambda)\right) \\
& \tilde{\mathbb{V}}^{+}\left(x_{0}, \lambda, \mu\right)=t^{-1}(\lambda) \operatorname{tr}_{a}\left(T_{a}^{+}\left(A, x_{0}, \lambda\right) r_{a b}(\lambda-\mu) \tilde{L}_{a}\left(x_{0}, \lambda\right) T_{a}^{-}\left(x_{0},-A, \lambda\right)\right) \\
& \tilde{\mathbb{V}}^{-}\left(x_{0}, \lambda, \mu\right)=t^{-1}(\lambda) \operatorname{tr}_{a}\left(T_{a}^{+}\left(A, x_{0}, \lambda\right) \tilde{L}_{a}\left(x_{0}, \lambda\right) r_{a b}(\lambda-\mu) T_{a}^{-}\left(x_{0},-A, \lambda\right)\right) .
\end{aligned}
$$

In order to identify the Lax pair associated to the Hamiltonian and momentum it is necessary to formulate the expansion of $\mathbb{V}$ in both negative and positive powers of $u$.

The first order contribution in the $u^{-1}$ expansion of the bulk $\mathbb{V}^{ \pm}$operator (we have self-explanatorily set the second spectral parameter $\left.v \equiv e^{\mu}\right)$ reads:

$$
\mathbb{V}^{ \pm(1)}=\frac{\beta^{2}}{8}\left(\frac{\beta}{2 m} \sigma^{z}\left(\pi^{ \pm}+\phi^{ \pm^{\prime}}\right)+i v\left(\sigma^{-} e^{-\frac{i \beta}{2} \phi^{ \pm}}-\sigma^{+} e^{\frac{i \beta}{2} \phi^{ \pm}}\right)\right)
$$

The first order contribution in the $u$ expansion reads:

$$
\hat{\mathbb{V}}^{ \pm(1)}=\frac{\beta^{2}}{8}\left(\frac{\beta}{2 m} \sigma^{z}\left(\pi^{ \pm}-\phi^{ \pm^{\prime}}\right)-i v^{-1}\left(\sigma^{-} e^{\frac{i \beta}{2} \phi^{ \pm}}-\sigma^{+} e^{-\frac{i \beta}{2} \phi^{ \pm}}\right)\right)
$$

Subtracting these two expressions and multiplying by $-\frac{2 i m}{\beta^{2}}$ we obtain the time component of the Lax pair associated to the Hamiltonian: $\left(\Omega^{ \pm}=e^{\frac{i \beta}{4} \phi^{ \pm} \sigma^{z}}\right)$

$$
\mathbb{V}_{\mathcal{H}}^{ \pm}=\frac{\beta}{4 i} \phi^{ \pm^{\prime}} \sigma^{z}+\frac{v m}{4 i} \Omega^{ \pm} \sigma^{y}\left(\Omega^{ \pm}\right)^{-1}+\frac{v^{-1} m}{4 i}\left(\Omega^{ \pm}\right)^{-1} \sigma^{y} \Omega^{ \pm}
$$

Adding now (3.27), (3.28), after multiplying with $-\frac{2 i m}{\beta^{2}}$, provides the time component of the Lax pair associated to the momentum:

$$
\mathbb{V}_{\mathcal{P}}^{ \pm}=\frac{\beta}{4 i} \pi^{ \pm} \sigma^{z}+\frac{v m}{4 i} \Omega^{ \pm} \sigma^{y}\left(\Omega^{ \pm}\right)^{-1}-\frac{v^{-1} m}{4 i}\left(\Omega^{ \pm}\right)^{-1} \sigma^{y} \Omega^{ \pm}
$$

The next step is the derivation of the relevant Lax pairs for the defect point from the left and the right, based on the expression (3.26). Indeed, after some cumbersome but quite straightforward computations, and after we have defined:

$$
w^{ \pm}=-\frac{i \beta}{m} \mathfrak{f}^{ \pm}, \quad \hat{w}^{ \pm}=\frac{i \beta}{m} \hat{\mathfrak{f}}^{ \pm},
$$

we conclude from the expansion in powers of $u^{-1}$ :

$$
\begin{aligned}
\tilde{\mathbb{V}}^{+(1)}= & \frac{i \beta^{2}}{8} \mathcal{D}^{-2} \sigma^{z}\left[w^{+}+w^{-}+e^{\frac{i \beta}{2} \phi^{-}} V a+e^{-\frac{i \beta}{2} \phi^{-}} V^{-1} \bar{a}\right] \\
& +\frac{i \beta^{2}}{4} \mathcal{D}^{-1} v\left[\sigma^{-} e^{-\frac{i \beta}{4}\left(\phi^{+}+\phi^{-}\right)} V^{-1}-\sigma^{+} e^{\frac{i \beta}{4}\left(\phi^{+}+\phi^{-}\right)} V\right],
\end{aligned}
$$


whereas the expansion in powers of $u$ leads to:

$$
\begin{aligned}
\hat{\tilde{\mathbb{V}}}^{+(1)}= & -\frac{i \beta^{2}}{8} \mathcal{D}^{-2} \sigma^{z}\left[\hat{w}^{+}+\hat{w}^{-}-e^{\frac{i \beta}{2} \phi^{-}} V \bar{a}-e^{-\frac{i \beta}{2} \phi^{-}} V^{-1} a\right] \\
& -\frac{i \beta^{2}}{4} \mathcal{D}^{-1} v^{-1}\left[\sigma^{-} e^{\frac{i \beta}{4}\left(\phi^{+}+\phi^{-}\right)} V-\sigma^{+} e^{-\frac{i \beta}{4}\left(\phi^{+}+\phi^{-}\right)} V^{-1}\right]
\end{aligned}
$$

Similarly, the corresponding expressions for $\tilde{\mathbb{V}}^{-(1)}, \tilde{\mathbb{V}}^{-(1)}$ are given below:

$$
\begin{aligned}
\tilde{\mathbb{V}}^{-(1)}= & \frac{i \beta^{2}}{8} \mathcal{D}^{-2} \sigma^{z}\left[w^{+}+w^{-}-e^{\frac{i \beta}{2} \phi^{+}} V^{-1} a-e^{-\frac{i \beta}{2} \phi^{+}} V \bar{a}\right] \\
& +\frac{i \beta^{2}}{4} \mathcal{D}^{-1} v\left[\sigma^{-} e^{-\frac{i \beta}{4}\left(\phi^{+}+\phi^{-}\right)} V-\sigma^{+} e^{\frac{i \beta}{4}\left(\phi^{+}+\phi^{-}\right)} V^{-1}\right] \\
\hat{\tilde{\mathbb{V}}}^{-(1)}=- & \frac{i \beta^{2}}{8} \mathcal{D}^{-2} \sigma^{z}\left[\hat{w}^{+}+\hat{w}^{-}+e^{\frac{i \beta}{2} \phi^{+}} V^{-1} \bar{a}+e^{-\frac{i \beta}{2} \phi^{+}} V a\right] \\
& -\frac{i \beta^{2}}{4} \mathcal{D}^{-1} v^{-1}\left[\sigma^{-} e^{\frac{i \beta}{4}\left(\phi^{+}+\phi^{-}\right)} V^{-1}-\sigma^{+} e^{-\frac{i \beta}{4}\left(\phi^{+}+\phi^{-}\right)} V\right] .
\end{aligned}
$$

We are now in a position to apply the scheme elaborated in [1]. The first manifest observation from the continuity conditions

$$
\begin{array}{rlrl}
\tilde{\mathbb{V}}^{+(1)}\left(x_{0}\right) & \rightarrow \mathbb{V}^{+(1)}\left(x_{0}^{+}\right), & x_{0}^{+} \rightarrow x_{0} \\
\tilde{\mathbb{V}}^{-(1)}\left(x_{0}\right) \rightarrow \mathbb{V}^{-(1)}\left(x_{0}^{-}\right), & x_{0}^{-} \rightarrow x_{0}
\end{array}
$$

(similar continuity conditions apply for the "hatted" quantities, but are omitted for brevity), is that:

$$
V=e^{\frac{i \beta}{4}\left(\phi^{+}-\phi^{-}\right)}
$$

and will be hereafter denoted as "first sewing condition $S_{1}$ ". Remember that from the very beginning one has already fixed the Casimir $C_{0}$ to some value $c_{0}$ independently of any sewing requirement. This can be seen as an "order zero condition $S_{0}$ " without any dependance in the bulk variables and yields a first-class constraint Poisson-commuting with all dynamical variables.

After imposing (3.37) the time components of the Lax pairs on the defect point take the following simple expressions:

$$
\tilde{\mathbb{V}}^{ \pm(1)}=\frac{\beta^{2}}{8}\left(\frac{\beta}{4 m} \sigma^{z}\left(\pi^{+}+\phi^{+^{\prime}}+\pi^{-}+\phi^{-^{\prime}}\right) \pm \frac{i \sigma^{z}}{4} \mathbb{M}+i v\left(\sigma^{-} e^{-\frac{i \beta}{2} \phi^{ \pm}}-\sigma^{+} e^{\frac{i \beta}{2} \phi^{ \pm}}\right)\right)
$$

and the first term in the $u$ expansion provides:

$$
\hat{\tilde{\mathbb{V}}}^{ \pm}=\frac{\beta^{2}}{8}\left(\frac{\beta}{4 m} \sigma^{z}\left(\pi^{+}-{\phi^{+}}^{\prime}+\pi^{-}-{\phi^{-}}^{\prime}\right) \pm \frac{i \sigma^{z}}{4} \hat{\mathbb{M}}-i v^{-1}\left(\sigma^{-} e^{\frac{i \beta}{2} \phi^{ \pm}}-\sigma^{+} e^{-\frac{i \beta}{2} \phi^{ \pm}}\right)\right)
$$

where we define:

$$
\begin{aligned}
& \mathbb{M}=e^{-\frac{i \beta}{4}\left(\phi^{+}+\phi^{-}\right)} \bar{a}+e^{\frac{i \beta}{4}\left(\phi^{+}+\phi^{-}\right)} a \\
& \hat{\mathbb{M}}=e^{\frac{i \beta}{4}\left(\phi^{+}+\phi^{-}\right)} \bar{a}+e^{-\frac{i \beta}{4}\left(\phi^{+}+\phi^{-}\right)} a
\end{aligned}
$$


Continuity conditions on the Lax pair as also described in (3.36) give rise to the following sewing conditions on the defect point $x_{0}$ associated to the momentum and the Hamiltonian respectively:

$$
\begin{array}{ll}
S_{2}: & \pi^{+}\left(x_{0}\right)-\pi^{-}\left(x_{0}\right)=\frac{i m}{\beta} \cos \frac{\beta}{4}\left(\phi^{+}\left(x_{0}\right)+\phi^{-}\left(x_{0}\right)\right)(a+\bar{a}) \\
S_{2}^{\prime}: & \phi^{+^{\prime}}\left(x_{0}\right)-{\phi^{-}}^{\prime}\left(x_{0}\right)=\frac{m}{\beta} \sin \frac{\beta}{4}\left(\phi^{+}\left(x_{0}\right)+\phi^{-}\left(x_{0}\right)\right)(\bar{a}-a)
\end{array}
$$

the prime denotes the derivative with respect to $x$.

It is instructive to point out that comparison of the extracted charges (3.24), (3.25), and the latter equations (3.41) with similar results obtained for instance in [13,14] reveal manifest discrepancies. We shall further comment on this matter in the discussion section.

Consistency of the sewing conditions $S_{1}, S_{2}, S_{2}^{\prime}$ can now be checked by computing their Poisson brackets with the first two Hamiltonians $\mathcal{H}, \mathcal{P}$. Indeed one gets:

$$
\begin{aligned}
\left\{\mathcal{H}, S_{1}\right\} & =-\frac{i \beta}{4}\left(\pi^{+}\left(x_{0}\right)-\pi^{-}\left(x_{0}\right)\right) S_{1}+\frac{i \beta}{4} S_{2} V+o(\mathcal{D}-2) \\
\left\{\mathcal{P}, S_{1}\right\} & =-\frac{i \beta}{4}\left(\phi^{+^{\prime}}\left(x_{0}\right)-\phi^{-^{\prime}}\left(x_{0}\right)\right) S_{1}+\frac{i \beta}{4} S_{2}^{\prime} V+o(\mathcal{D}-2)
\end{aligned}
$$

We recall that on-shell $\mathcal{D} \approx 2 ; \mathcal{A} \approx 0$.

Consider now the Poisson brackets of $\mathcal{H}, \mathcal{P}$ with $S_{2}, S_{2}^{\prime}$. One easily obtains that they are given by expressions of the following form:

$$
\begin{aligned}
& \left\{\mathcal{P}, S_{2}\right\} \\
& =\left(\pi^{+^{\prime}}\left(x_{0}\right)-\pi^{-^{\prime}}\left(x_{0}\right)\right) F\left(\pi^{+}\left(x_{0}\right)+\pi^{-}\left(x_{0}\right),{\phi^{+}}^{\prime}\left(x_{0}\right)+{\phi^{-{ }^{\prime}}}\left(x_{0}\right), \phi^{+}\left(x_{0}\right), \phi^{-}\left(x_{0}\right), V, a, \bar{a}\right) \\
& \left\{\mathcal{H}, S_{2}\right\} \\
& =\left(\phi^{+^{\prime \prime}}\left(x_{0}\right)-\phi^{-^{\prime \prime}}\left(x_{0}\right)\right) G\left(\pi^{+}\left(x_{0}\right)+\pi^{-}\left(x_{0}\right), \phi^{+^{\prime}}\left(x_{0}\right)+\phi^{{ }^{\prime}}\left(x_{0}\right), \phi^{+}\left(x_{0}\right), \phi^{-}\left(x_{0}\right), V, a, \bar{a}\right)
\end{aligned}
$$

where $F$ and $G$ are given functions to be computed specifically. Poisson brackets with $S_{2}^{\prime}$ are given by similar expressions exchanging $\mathcal{H}$ and $\mathcal{P}$. Note that (contrary to the non-linear Schroedinger case) no term proportional to the singular contribution $\delta(0)$ arise, they fully cancel in the Poisson brackets. It is therefore to be expected that the finite terms on the r.h.s. of both PB's will yield the third sewing conditions $S_{3}, S_{3}^{\prime}$ which will respectively take the form (expected from general arguments) $\left(\pi^{+^{\prime}}\left(x_{0}\right)-\pi^{-^{\prime}}\left(x_{0}\right)\right)=-F$ and $\left(\phi^{+^{\prime \prime}}\left(x_{0}\right)-\phi^{-{ }^{\prime \prime}}\left(x_{0}\right)=-G\right.$. Explicit derivation of these sewing conditions from higher terms in the expansion of the

$\mathbb{V}$ operators is technically quite cumbersome but we conjecture that they will coincide with the r.h.s. of (3.43).

Let us now reconsider the Poisson bracket $\{\mathcal{H}, \mathcal{P}\}$. It turns out from explicit computations that one has in fact:

$$
\{\mathcal{H}, \mathcal{P}\} \approx 0,
$$

i.e. the Poisson bracket vanishes provided that the constraints $S_{1}, S_{2}, S_{2}^{\prime}$ be satisfied. 
Assuming that the Hamiltonians $\mathcal{H}$ and $\mathcal{P}$ weakly preserve all constraints (as already established for $S_{1}$ and conjectured for $S_{2}, S_{2}^{\prime}$ ) we deduce that the momentum and Hamiltonian Dirac commute. Our construction of Type IIa defect is thus compatible with a statement of Liouville-integrability on-shell.

Type-IIb defect. Having completed the basic computations regarding the type-IIa defect we now introduce the $\tilde{L}$ matrix relevant to the type-IIb defect. In fact, the new defect matrix arises via a simple matrix multiplication $\tilde{L}\left(x_{0}, \lambda\right) \rightarrow \sigma^{x} \tilde{L}\left(x_{0}, \lambda\right)$ :

$$
\tilde{L}(\lambda)=\left(\begin{array}{cc}
a & e^{\lambda} V^{-1}-e^{-\lambda} V \\
e^{\lambda} V-e^{-\lambda} V^{-1} & \bar{a}
\end{array}\right) .
$$

where $\tilde{L}$ is given in (3.2). It is clear that the defect algebra (3.3) is valid in this case as well due to the property that $\sigma^{x} \otimes \sigma^{x}$ commutes with the $r$-matrix. Following the same process as in the case of type-IIa defect we are able to extract the first integrals of motion. Recalling the expression for the generating function of integrals of motion, introduced in the previous example, we conclude that

$$
\begin{aligned}
I^{(1)}= & -\frac{m}{4 i} \int_{-L}^{x_{0}^{-}} d x\left(-\frac{\beta^{2}}{2 m^{2}} \mathfrak{f}^{-2}(x)+\cos \left(\beta \phi^{-}(x)\right)\right)-\frac{m}{4 i} \int_{x_{0}^{+}}^{L} d x\left(-\frac{\beta^{2}}{2 m^{2}} \mathfrak{f}^{+2}(x)+\cos \left(\beta \phi^{+}(x)\right)\right) \\
& +\frac{i}{\hat{\mathcal{D}}}\left(e^{\frac{i \beta}{4}\left(\phi^{+}\left(x_{0}\right)-\phi^{-}\left(x_{0}\right)\right)} \bar{a}+e^{-\frac{i \beta}{4}\left(\phi^{+}\left(x_{0}\right)-\phi^{-}\left(x_{0}\right)\right)} a\right)-\frac{\beta}{2 m \hat{\mathcal{D}}}\left(\mathfrak{f}^{+}\left(x_{0}\right)-\mathfrak{f}^{-}\left(x_{0}\right)\right) \hat{\mathcal{A}}
\end{aligned}
$$

where we define:

$$
\begin{aligned}
& \hat{\mathcal{D}}=e^{\frac{i \beta}{4}\left(\phi^{+}\left(x_{0}\right)+\phi^{-}\left(x_{0}\right)\right)} V-e^{-\frac{i \beta}{4}\left(\phi^{+}\left(x_{0}\right)+\phi^{-}\left(x_{0}\right)\right)} V^{-1}, \\
& \hat{\mathcal{A}}=e^{\frac{i \beta}{4}\left(\phi^{+}\left(x_{0}\right)+\phi^{-}\left(x_{0}\right)\right)} V+e^{-\frac{i \beta}{4}\left(\phi^{+}\left(x_{0}\right)+\phi^{-}\left(x_{0}\right)\right)} V^{-1} .
\end{aligned}
$$

If we now perform the same kind of expansion but for $\lambda \rightarrow-\infty$, we end up with a similar expression as in the Type IIa case, by simply exploiting the fundamental symmetry of the monodromy matrix (3.21):

$$
\begin{aligned}
I^{(-1)}= & \frac{m}{4 i} \int_{-L}^{x_{0}^{-}} d x\left(-\frac{\beta^{2}}{2 m^{2}} \hat{\mathfrak{f}}^{-2}(x)+\cos \left(\beta \phi^{-}(x)\right)\right)-\frac{m}{4 i} \int_{x_{0}^{+}}^{L} d x\left(-\frac{\beta^{2}}{2 m^{2}} \hat{\mathfrak{f}}^{+2}(x)+\cos \left(\beta \phi^{+}(x)\right)\right) \\
& +\frac{i}{\hat{\mathcal{D}}}\left(e^{-\frac{i \beta}{4}\left(\phi^{+}\left(x_{0}\right)-\phi^{-}\left(x_{0}\right)\right)} \bar{a}+e^{\frac{i \beta}{4}\left(\phi^{+}\left(x_{0}\right)+\phi^{-}\left(x_{0}\right)\right)} a\right)-\frac{\beta}{2 m \hat{\mathcal{D}}}\left(\hat{\mathfrak{f}}^{+}\left(x_{0}\right)-\hat{\mathfrak{f}}^{-}\left(x_{0}\right)\right) \hat{\mathcal{A}}
\end{aligned}
$$

The corresponding Hamiltonian then reads as:

$$
\begin{aligned}
\mathcal{H}= & \frac{2 i m}{\beta^{2}}\left(I^{(1)}-I^{(-1)}\right) \\
= & \int_{-L}^{x_{0}^{-}} d x\left(\frac{1}{2}\left(\pi^{-2}(x)+\phi^{-^{\prime} 2}(x)\right)-\frac{m^{2}}{\beta^{2}} \cos \left(\beta \phi^{-}(x)\right)\right) \\
& +\int_{x_{0}^{+}}^{L} d x\left(\frac{1}{2}\left(\pi^{+2}(x)+\phi^{+^{\prime}}(x)\right)-\frac{m^{2}}{\beta^{2}} \cos \left(\beta \phi^{+}(x)\right)\right) \\
& +\frac{4 m i}{\beta^{2} \hat{\mathcal{D}}} \sin \frac{\beta}{4}\left(\phi^{+}\left(x_{0}\right)-\phi^{-}\left(x_{0}\right)\right)(a-\bar{a})-\frac{2 i}{\beta \hat{\mathcal{D}}}\left(\phi^{+^{\prime}}\left(x_{0}\right)-\phi^{-^{\prime}}\left(x_{0}\right)\right) \hat{\mathcal{A}}
\end{aligned}
$$


and we may also identify the momentum as:

$$
\begin{aligned}
\mathcal{P}= & \frac{2 i m}{\beta^{2}}\left(I^{(1)}+I^{(-1)}\right) \\
= & \int_{-L}^{x_{0}^{-}} d x \phi^{-^{\prime}}(x) \pi^{-}(x)+\int_{x_{0}^{+}}^{L} d x{\phi^{+}}^{\prime}(x) \pi^{+}(x) \\
& -\frac{4 m}{\beta^{2} \hat{\mathcal{D}}} \cos \frac{\beta}{4}\left(\phi^{+}\left(x_{0}\right)-\phi^{-}\left(x_{0}\right)\right)(\bar{a}+a)-\frac{2 i}{\beta \hat{\mathcal{D}}}\left(\pi^{+}\left(x_{0}\right)-\pi^{-}\left(x_{0}\right)\right) \hat{\mathcal{A}} .
\end{aligned}
$$

Similarly we may identify the time components of the Lax pairs associated to the charges $I^{(1)}, I^{(-1)}$. From the expansion in powers of $u^{-1}$ we have:

$$
\begin{aligned}
\tilde{\mathbb{V}}^{+(1)}= & \frac{i \beta^{2}}{8} \hat{\mathcal{D}}^{-2} \sigma^{z}\left[w^{-}-w^{+}+e^{\frac{i \beta}{2} \phi^{-}} V a+e^{-\frac{i \beta}{2} \phi^{-}} V^{-1} \bar{a}\right] \\
& +\frac{i \beta^{2}}{4} \hat{\mathcal{D}}^{-1} v\left[\sigma^{+} e^{\frac{i \beta}{4}\left(\phi^{+}-\phi^{-}\right)} V^{-1}+\sigma^{-} e^{-\frac{i \beta}{4}\left(\phi^{+}-\phi^{-}\right)} V\right]
\end{aligned}
$$

whereas the expansion in powers of $u$ leads to:

$$
\begin{aligned}
\hat{\tilde{\mathbb{V}}}+(1)= & \frac{i \beta^{2}}{8} \hat{\mathcal{D}}^{-2} \sigma^{z}\left[\hat{w}^{+}-\hat{w}^{-}+e^{\frac{i \beta}{2} \phi^{-}} V \bar{a}+e^{-\frac{i \beta}{2} \phi^{-}} V^{-1} a\right] \\
& +\frac{i \beta^{2}}{4} \hat{\mathcal{D}}^{-1} v^{-1}\left[\sigma^{+} e^{-\frac{i \beta}{4}\left(\phi^{+}-\phi^{-}\right)} V+\sigma^{-} e^{\frac{i \beta}{4}\left(\phi^{+}-\phi^{-}\right)} V^{-1}\right]
\end{aligned}
$$

Similarly, the corresponding expressions for $\tilde{\mathbb{V}}^{-(1)}, \tilde{\mathbb{V}}^{-(1)}$ are given below:

$$
\begin{aligned}
\tilde{\mathbb{V}}^{-(1)}= & \frac{i \beta^{2}}{8} \hat{\mathcal{D}}^{-2} \sigma^{z}\left[w^{+}-w^{-}-e^{-\frac{i \beta}{2} \phi^{+}} V^{-1} a-e^{\frac{i \beta}{2} \phi^{+}} V \bar{a}\right] \\
& +\frac{i \beta^{2}}{4} \hat{\mathcal{D}}^{-1} v\left[\sigma^{+} e^{-\frac{i \beta}{4}\left(\phi^{+}-\phi^{-}\right)} V^{-1}+\sigma^{-} e^{\frac{i \beta}{4}\left(\phi^{+}-\phi^{-}\right)} V\right] \\
\hat{\tilde{\mathbb{V}}^{-}(1)=} & \frac{i \beta^{2}}{8} \hat{\mathcal{D}}^{-2} \sigma^{z}\left[\hat{w}^{+}-\hat{w}^{-}-e^{-\frac{i \beta}{2} \phi^{+}} V^{-1} \bar{a}-e^{\frac{i \beta}{2} \phi^{+}} V a\right] \\
& +\frac{i \beta^{2}}{4} \hat{\mathcal{D}}^{-1} v^{-1}\left[\sigma^{-} e^{-\frac{i \beta}{4}\left(\phi^{+}-\phi^{-}\right)} V^{-1}+\sigma^{+} e^{\frac{i \beta}{4}\left(\phi^{+}-\phi^{-}\right)} V\right] .
\end{aligned}
$$

The first manifest observation from the continuity conditions

$$
\begin{aligned}
\tilde{\mathbb{V}}^{+(1)}\left(x_{0}\right) \rightarrow \mathbb{V}^{+(1)}\left(x_{0}^{+}\right), & x_{0}^{+} \rightarrow x_{0} \\
\tilde{\mathbb{V}}^{-(1)}\left(x_{0}\right) \rightarrow \mathbb{V}^{-(1)}\left(x_{0}^{-}\right), & x_{0}^{-} \rightarrow x_{0}
\end{aligned}
$$

(similar continuity conditions apply for the "hatted" quantities as well), is that:

$$
\tilde{S}_{1}: \quad V=i e^{-\frac{i \beta}{4}\left(\phi^{+}+\phi^{-}\right)} .
$$

After imposing (3.56) the time components of the Lax pairs on the defect point take the following simple expressions:

$$
\tilde{\mathbb{V}}^{ \pm(1)}=\frac{\beta^{2}}{8}\left( \pm \frac{\beta}{4 m} \sigma^{z}\left(\pi^{+}+{\phi^{+}}^{\prime}-\pi^{-}-\phi^{-^{\prime}}\right)+\frac{\sigma^{z}}{4} \tilde{\mathbb{M}}+i v\left(\sigma^{-} e^{-\frac{i \beta}{2} \phi^{ \pm}}-\sigma^{+} e^{\frac{i \beta}{2} \phi^{ \pm}}\right)\right)
$$


and the first term in the $u$ expansion provides:

$$
\hat{\tilde{\mathbb{V}}}^{ \pm(1)}=\frac{\beta^{2}}{8}\left( \pm \frac{\beta}{4 m} \sigma^{z}\left(\pi^{+}-\phi^{+^{\prime}}-\pi^{-}+\phi^{-^{\prime}}\right)+\frac{\sigma^{z}}{4} \hat{\tilde{\mathbb{M}}}-i v^{-1}\left(\sigma^{-} e^{\frac{i \beta}{2} \phi^{ \pm}}-\sigma^{+} e^{-\frac{i \beta}{2} \phi^{ \pm}}\right)\right)
$$

where we define:

$$
\begin{aligned}
& \tilde{\mathbb{M}}=-e^{\frac{i \beta}{4}\left(\phi^{+}-\phi^{-}\right)} \bar{a}+e^{-\frac{i \beta}{4}\left(\phi^{+}-\phi^{-}\right)} a \\
& \hat{\tilde{M}}=e^{-\frac{i \beta}{4}\left(\phi^{+}-\phi^{-}\right)} \bar{a}-e^{\frac{i \beta}{4}\left(\phi^{+}-\phi^{-}\right)} a
\end{aligned}
$$

Continuity conditions on the Lax pair as also described in (3.55) give rise to the following sewing conditions on the defect point $x_{0}$ associated to the momentum and the Hamiltonian respectively:

$$
\begin{array}{lll}
\tilde{S}_{2}: & \phi^{+^{\prime}}\left(x_{0}\right)+\phi^{-^{\prime}}\left(x_{0}\right)=\frac{m}{\beta} \cos \frac{\beta}{4}\left(\phi^{+}\left(x_{0}\right)-\phi^{-}\left(x_{0}\right)\right)(a-\bar{a}) \\
\tilde{S}_{2}^{\prime}: & \pi^{+}\left(x_{0}\right)+\pi^{-}\left(x_{0}\right)=-\frac{i m}{\beta} \sin \frac{\beta}{4}\left(\phi^{+}\left(x_{0}\right)-\phi^{-}\left(x_{0}\right)\right)(\bar{a}+a)
\end{array}
$$

The energy and momentum are again in weak involution that is:

$$
\{\mathcal{H}, \mathcal{P}\} \approx 0
$$

provided that the constraints $\tilde{S}_{1}, \tilde{S}_{2}, \tilde{S}_{2}^{\prime}$ are satisfied.

Similar consistency conditions as (3.42), (3.43) will occur between the charges and the sewing conditions. They shall be omitted here however for the sake of brevity. Hence, assuming that all higher sewing conditions are also weakly conserved by the momentum and Hamiltonian we have established that the momentum and Hamiltonian Dirac commute and the Type IIb defect is Liouville integrable on-shell.

Equations of motion. To extract the associated equations of motion for the left and right bulk theories as well as the defect point one needs to employ the zero curvature condition expressed as:

$$
\dot{\mathbb{U}}^{ \pm}(x, t)-\mathbb{V}^{ \pm^{\prime}}(x, t)+\left[\mathbb{U}^{ \pm}(x, t), \mathbb{V}^{ \pm}(x, t)\right]=0 \quad x \neq x_{0}
$$

As usual the dot denotes derivative with respect to $t$.

On the defect point in particular the zero curvature condition is formulated as (this is also transparent when discussing the continuum limit of discrete theories (see e.g. [1])

$$
\dot{\tilde{L}}\left(x_{0}\right)=\tilde{\mathbb{V}}^{+}\left(x_{0}\right) \tilde{L}\left(x_{0}\right)-\tilde{L}\left(x_{0}\right) \tilde{\mathbb{V}}^{-}\left(x_{0}\right),
$$

and describes explicitly the jump occurring across the defect point.

The equations of motion are obtained via the zero curvature conditions as described above or (equivalently thanks to the sewing conditions) via the Hamiltonian equations i.e.

$$
\begin{aligned}
\dot{\phi}^{ \pm} & =\left\{\mathcal{H}, \phi^{ \pm}\right\}, & \dot{\pi}^{ \pm} & =\left\{\mathcal{H}, \pi^{ \pm}\right\}, \\
\dot{\mathrm{e}} & =\{\mathcal{H}, \mathrm{e}\}, & \mathrm{e} & \in\{a, \bar{a}, V\}
\end{aligned}
$$


bear also in mind that

$$
\left\{\pi^{ \pm}, \mathrm{e}\right\}=\left\{\phi^{ \pm}, \mathrm{e}\right\}=0 .
$$

For the left and right bulk theories the familiar equations of motion for the sine-Gordon model arise

$$
\ddot{\phi}^{ \pm}(x, t)-\phi^{ \pm^{\prime \prime}}(x, t)+\frac{m^{2}}{\beta} \sin \left(\beta \phi^{ \pm}(x, t)\right)=0
$$

On the defect point the time evolution of the defect degrees of freedom for the type-IIa defect are found to be:

$$
\begin{aligned}
& \dot{a}=-\frac{m}{2 \mathcal{D}^{2}} \mathcal{A} a \cos \frac{\beta}{4}\left(\phi^{+}+\phi^{-}\right)(\bar{a}-a)-\frac{m}{\mathcal{D}} \cos \frac{\beta}{4}\left(\phi^{+}+\phi^{-}\right)\left(V^{2}-V^{-2}\right)
\end{aligned}
$$

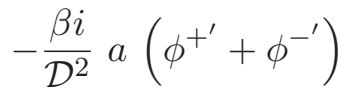

$$
\begin{aligned}
& \dot{\bar{a}}=\frac{m}{2 \mathcal{D}^{2}} \mathcal{A} \bar{a} \cos \frac{\beta}{4}\left(\phi^{+}+\phi^{-}\right)(\bar{a}-a)-\frac{m}{\mathcal{D}} \cos \frac{\beta}{4}\left(\phi^{+}+\phi^{-}\right)\left(V^{2}-V^{-2}\right) \\
& +\frac{i \beta}{\mathcal{D}^{2}} \bar{a}\left(\phi^{+^{\prime}}+\phi^{-^{\prime}}\right) \\
& \dot{V}=\frac{m}{2 \mathcal{D}} V \cos \left(\frac{\beta}{4}\left(\phi^{+}+\phi^{-}\right)\right)(a+\bar{a}) .
\end{aligned}
$$

Similarly, the time evolution of the degrees of freedom for the type-IIb defect are gives as:

$$
\begin{aligned}
\dot{a}= & \frac{i m}{2 \hat{\mathcal{D}}^{2}} a \hat{\mathcal{A}} \sin \frac{\beta}{4}\left(\phi^{+}-\phi^{-}\right)(a-\bar{a})-\frac{i m}{\hat{\mathcal{D}}} \sin \frac{\beta}{4}\left(\phi^{+}-\phi^{-}\right)\left(V^{2}-V^{-2}\right) \\
& -\frac{i \beta}{\hat{\mathcal{D}}^{2}} a\left(\phi^{+^{\prime}}-\phi^{-^{\prime}}\right) \\
\dot{\bar{a}}= & -\frac{i m}{2 \hat{\mathcal{D}}^{2}} \bar{a} \hat{\mathcal{A}} \sin \frac{\beta}{4}\left(\phi^{+}-\phi^{-}\right)-\frac{i m}{\hat{\mathcal{D}}} \sin \frac{\beta}{4}\left(\phi^{-}-\phi^{-}\right)\left(V^{2}-V^{-2}\right) \\
& +\frac{i \beta}{\hat{\mathcal{D}}^{2}} \bar{a}\left({\phi^{+}}^{\prime}-\phi^{-^{\prime}}\right) \\
\dot{V}= & \frac{i m}{2 \hat{\mathcal{D}}} \sin \frac{\beta}{4}\left(\phi^{+}-\phi^{-}\right) V(a+\bar{a})
\end{aligned}
$$

Let us end our construction of the sine Gordon defect theory with a few comments and discussions on some tricky issues which have arisen in the course of our presentation.

\section{Discussion}

Comparison of our expressions for the charges, for both defects IIa and IIb, with the corresponding findings appearing in e.g. [13, 14, 23, 24] leads in this case to discrepancies. We believe this to be due to the basic differences in the particular methodologies adopted. We have already dwelt on the off-shell $\rightarrow$ on-shell approach which we advocate. By contrast in $[13,14,23,24]$, the degrees of freedom associated to the defect are not present as independent dynamical variables in the whole construction, but they are a priori related 
to the fields of the right and left theories and their derivatives. This is one key difference compared to our approach, where only at the very end are the "off-shell" degrees of freedom of the defect related to the left and right limit of bulk dynamical variables, and this specifically through the sewing conditions.

Moreover and even more to the point, in the course pursued in [14, 23, 24] the conservation of the charges is shown via the zero curvature condition. This means that the conservation of charges is proved only for a single Hamiltonian evolution (out of the hierarchy of such) corresponding to the particular choice of the $\mathbb{V}$ operator. However no underlying Poisson structure is available hence the involution of the charges cannot be proven which would make Liouville integrability manifest.

Let us be more specific: In $[14,23,24]$ one observes that in the presence of defects the construction of the defect matrix, and the proof of the conservation of the charges are based on two fundamental equations:

$$
\begin{aligned}
& \dot{\tilde{L}}\left(x_{0}\right)=\tilde{\mathbb{V}}^{+}\left(x_{0}\right) \tilde{L}\left(x_{0}\right)-\tilde{L}\left(x_{0}\right) \tilde{\mathbb{V}}^{-}\left(x_{0}\right) \\
& \tilde{L}^{\prime}\left(x_{0}\right)=\mathbb{U}^{+}\left(x_{0}\right) \tilde{L}\left(x_{0}\right)-\tilde{L}\left(x_{0}\right) \mathbb{U}^{-}\left(x_{0}\right) .
\end{aligned}
$$

Equation (4.1) is a "time" evolution of the $\tilde{L}\left(x_{0}\right)$ matrix, and arises alternatively in our Hamiltonian description from application of the second conserved Hamiltonian using the canonical Poisson structure (see equation (3.64)).

Equation (4.2) is a second "time" evolution of the $\tilde{L}\left(x_{0}\right)$ matrix, and should naturally emerge alternatively in our Hamiltonian description from application of the first conserved Hamiltonian using the canonical Poisson structure (see 3.63). Remember that in the Hamiltonian approach to Liouville integrability all "times" associated to the respective Hamiltonians of the hierarchy are equivalent.

It follows that such equations as (4.1), (4.2) are automatically present in our derivation but only as consequences of the basic procedure. The reciprocal statement may not be true. Compatibility of two "time" evolutions a priori does not guarantee the existence of a Poisson structure which would render these two evolutions Hamiltonian. Were such a Poisson structure built, it only guarantees the Poisson commutation of the two corresponding Hamiltonians but not the existence of higher conserved Poisson commuting Hamiltonians, unless a Magri-type algorithm [26, 27] allows to build a recursion operator from the two Hamiltonians and the Poisson structure, and hence to deduce the hierarchy of Poisson structure dual to the postulated hierarchy of hamiltonians. To formulate in another language: Equation (4.2) can be interpreted indeed as a Bäcklund transformation (see e.g. $[23,24]$ ) acting on the defect configuration by "space translation"; ${ }^{1}$ these transformations do act on the space of configurations as a group of dressing transformations; but no Lie-Poisson structure of this group is manifest.

This second formulation thus yields a weaker form of integrability, which may be characterized as "Lax integrability" or "algebraic integrability" or even "Lagrangian integrability". And as we have now seen, Liouville-integrable defects are always Lax-integrable; the

\footnotetext{
${ }^{1}$ We wish to thank the referee for pointing out this interpretation to us
} 
reciprocal is not true and there are more Lax-integrable defects than Liouville-integrable defects. The discrepancies occurring in the SG case are thus not unexpected.

To conclude on this point: in our approach integrability is by construction ensured even in the presence of the defect, due to the fact that the defect matrix $\tilde{L}$ satisfies the same fundamental quadratic algebra, as the one the monodromy matrices $T^{ \pm}$satisfies. One more key ingredient is apparent via the proposed methodology, that is the systematic construction of the time component of the Lax pair, which eventually leads to non-trivial gluing condition among the degrees of freedom of the defect and the right-left fields and their derivatives. The whole process is consistent and is based on first principles, hence no further assumptions or ad hoc formulations are required. Moreover, various consistency checks have been performed (see relevant previous works $[1,22]$ ) especially in comparison with the corresponding discrete description [22] to guarantee the validity of the adopted process.

Note that if the defect is movable then possibly there exists some Bäcklund transformation associated, although this is not a priori clear. Nevertheless, still the question of the relevant Poisson structure is raised. Another issue raised is about dressing and its compatibility with the Poisson structure, in other words is the dressing/Bäcklund transformation group a Lie Poisson group? This is true usually in the bulk case, but not obvious in the defect case.

A final comment is of order. As indicated in the discussion the Poisson commutation of the bulk-plus-defect Hamiltonians is not verified off-shell in the sine Gordon case (contrary to the NLS case). We expect that this is due to the occurrence, in the derivation of Poisson brackets between these off-shell defect Hamiltonians, of Poisson structures which are for all instance and purposes distributions (delta terms). Thus the need for regularizations arises (for instance by discretization, see e.g. [1, 22]), hence the possibility of hampering integrability off-shell.

One may expect that this would also occur on-shell, but it does not seem to be the case at least on the SG example. This may in fact be intrinsically related to the definition of the sewing conditions as being the analytic conditions which allow to exactly identify the Hamiltonian-induced equations of motion with the Lax equations, once the Lax partner $\mathbb{V}$ is built according to the fundamental pattern a la Semenov-Tjan-Shanskii [30].

What may happen systematically in this procedure (and possibly only when ultra-local Poisson structures are involved) is that if breaking of integrability occurs off-shell, it can only occur through precisely these singular terms, which are killed by the sewing conditions and Liouville integrability is therefore reestablished on-shell.

\section{Acknowledgments}

This work was partially supported by CNRS, Université de Cergy Pontoise, and ANR Project DIADEMS (Programme Blanc ANR SIMI 1 2010-BLAN-0120-02). J.A. wishes to thank Department of Engineering, Patras University, and A.D. thanks UCP and LPTM Cergy, for ongoing mutual hospitality. 
Open Access. This article is distributed under the terms of the Creative Commons Attribution License which permits any use, distribution and reproduction in any medium, provided the original author(s) and source are credited.

\section{References}

[1] Avan, Liouville integrable defects: the non-linear Schrödinger paradigm, JHEP 01 (2012) 040 [arXiv:1110.4728] [INSPIRE].

[2] G. Delfino, G. Mussardo and P. Simonetti, Statistical models with a line of defect, Phys. Lett. B 328 (1994) 123 [hep-th/9403049] [InSPIRE].

[3] G. Delfino, G. Mussardo and P. Simonetti, Scattering theory and correlation functions in statistical models with a line of defect, Nucl. Phys. B 432 (1994) 518 [hep-th/9409076] [INSPIRE].

[4] E. Corrigan and C. Zambon, A Transmission matrix for a fused pair of integrable defects in the sine-Gordon model, J. Phys. A 43 (2010) 345201 [arXiv:1006.0939] [InSPIRE].

[5] R. Konik and A. LeClair, Purely transmitting defect field theories, Nucl. Phys. B 538 (1999) 587 [hep-th/9703085].

[6] P. Bowcock, E. Corrigan and C. Zambon, Some aspects of jump-defects in the quantum sine-Gordon model, JHEP 08 (2005) 023 [hep-th/0506169] [INSPIRE].

[7] F. Nemes, Semiclassical analysis of defect sine-Gordon theory, Int. J. Mod. Phys. A 25 (2010) 4493 [arXiv:0909.3268] [InSPIRE].

[8] E. Corrigan and C. Zambon, Comments on defects in the $a_{r}$ Toda field theories, J. Phys. A 42 (2009) 304008 [arXiv:0902.1307] [InSPIRE].

[9] P. Bowcock, E. Corrigan and C. Zambon, Affine Toda field theories with defects, JHEP 01 (2004) 056 [hep-th/0401020] [INSPIRE].

[10] E. Corrigan and C. Zambon, On purely transmitting defects in affine Toda field theory, JHEP 07 (2007) 001 [arXiv:0705.1066] [INSPIRE].

[11] E. Corrigan and C. Zambon, A New class of integrable defects, J. Phys. A 42 (2009) 475203 [arXiv:0908.3126] [INSPIRE].

[12] E. Corrigan and C. Zambon, Jump-defects in the nonlinear Schrodinger model and other non-relativistic field theories, Nonlinearity 19 (2006) 1447 [nlin/0512038].

[13] I. Habibullin and A. Kundu, Quantum and classical integrable sine-Gordon model with defect, Nucl. Phys. B 795 (2008) 549 [arXiv:0709.4611] [INSPIRE].

[14] V. Caudrelier, On a Systematic Approach to Defects in Classical Integrable Field Theories, Int. J. Geom. Methods M. 5 (2008) 1085 [arXiv:0704.2326].

[15] Z. Bajnok and A. George, From defects to boundaries, Int. J. Mod. Phys. A 21 (2006) 1063 [hep-th/0404199] [INSPIRE].

[16] Z. Bajnok, Equivalences between spin models induced by defects, J. Stat. Mech. 0606 (2006) P06010 [hep-th/0601107] [INSPIRE].

[17] Z. Bajnok and Z. Simon, Solving topological defects via fusion, Nucl. Phys. B 802 (2008) 307 [arXiv:0712.4292] [INSPIRE]. 
[18] R. Weston, An Algebraic Setting for Defects in the XXZ and sine-Gordon Models, arXiv:1006.1555 [INSPIRE].

[19] M. Mintchev, É. Ragoucy and P. Sorba, Scattering in the presence of a reflecting and transmitting impurity, Phys. Lett. B 547 (2002) 313 [hep-th/0209052] [INSPIRE].

[20] M. Mintchev, É. Ragoucy and P. Sorba, Reflection transmission algebras, J. Phys. A 36 (2003) 10407 [hep-th/0303187] [InSPIRE].

[21] V. Caudrelier, M. Mintchev and É. Ragoucy, The Quantum non-linear Schrödinger model with point-like defect, J. Phys. A 37 (2004) L367 [hep-th/0404144] [INSPIRE].

[22] Doikou, Defects in the discrete non-linear Schrödinger model, Nucl. Phys. B 854 (2012) 153 [arXiv: 1106.1602] [INSPIRE].

[23] A. Aguirre, T. Araujo, J. Gomes and A. Zimerman, Type-II Bácklund Transformations via Gauge Transformations, JHEP 12 (2011) 056 [arXiv:1110.1589] [INSPIRE].

[24] A.R. Aguirre, Inverse scattering approach for massive Thirring models with integrable type-II defects, J. Phys. A 45 (2012) 205205 [arXiv:1111.5249] [inSPIRE].

[25] J.M. Maillet, New integrable canonical structures in two-dimensional models, Nucl. Phys. B 269 (1986) 54 [inSPIRE].

[26] F. Magri, P. Casati, G. Falqui and M. Pedroni, Eight Lectures on Integrable Systems, Lect. Notes Phys. 638 (2004) 209.

[27] W. Oevel and O. Ragnisco, R-matrices and higher poisson brackets for integrable systems, Physica A 161 (1989) 181.

[28] J. Avan and E. Ragoucy, Rational Calogero-Moser model: explicit form and R-matrix of the second Poisson structure, SIGMA 8 (2012) 079 [arXiv:1207.5368].

[29] L.D. Faddeev and L.A. Takhtakajan, Hamiltonian Methods in the Theory of Solitons, Springer-Verlag (1987).

[30] M. Semenov-Tian-Shansky, What is a classical r-matrix?, Funct. Anal. Appl. 17 (1983) 259 [INSPIRE].

[31] M. Jimbo, Quantum R matrix for the generalized Toda system, Commun. Math. Phys. 102 (1986) 537 [http://projecteuclid.org/euclid.cmp/1104114539].

[32] Avan, Boundary Lax pairs for the $A_{n}^{(1)}$ Toda field theories, Nucl. Phys. B 821 (2009) 481 [arXiv: 0809.2734] [INSPIRE]. 